\title{
Sécheresse et eaux souterraines
}

\author{
Jean Margat \\ B.R.G.M. - France
}

\begin{abstract}
La sécheresse étant définie comme une déficience conjoncturelle significative des précipitations - donc des apports d'eau météorique aux "systèmes d'eau» terrestres pendant une durée assez longue (saisonnière, annuelle, voire pluriannuelle) et sur une étendue assez vaste, par rapport aux moyennes (au moins pluri-décennales) ou aux occurrences médianes, ses relations avec les eaux souterraines sont à considérer de deux points de vue :

- celui de la sensibilité des eaux souterraines à ces carences d'apport et des conséquences de la sécheresse sur leur régime naturel ;

- et celui des contributions spontanées des eaux souterraines à l'atténuation des effets de la sécheresse sur les écoulements superficiels, ou encore de leur contribution artificielle par leur exploitation, à la compensation des manques des utilisateurs d'eau pluviale ou superficielle.
\end{abstract}

\begin{abstract}
Auparavant il est opportun de rappeler très brièvement quelques caractères distinctifs primordiaux des eaux souterraines par rapport aux autres eaux continentales.
\end{abstract}

\section{Les eaux souterraines: une triple variété}

Les « gisements » d'eau souterraine, les aquifères sont à la fois conducteurs et accumulateurs, aussi les eaux souterraines se définissent par des flux et par des stocks. Les caractéristiques qui commandent les uns et les autres sont en partie indépendantes, et les structures géologiques qui déterminent la géométrie des aquifères et leurs communications avec la surface sont extrêmement diverses. D'où trois sortes de variété de situation et de comportement des eaux souterraines :

\section{Droughts and underground water}

The effect of droughts on underground water are of two types : there is the effect on supply and flowing conditions of free waters, consequences for exploitation of the resource. The sensitivity of free water to supply deficiency resulting from drought (winter mainly but also summer in relation to the rainy season that it precedes : the reconstitution of the humidity of the soil will reduce efficient infiltrations) depends on the part taken by meteoric contribution in their supply and the flow/stock ratio of each waterway.

Droughts reduce the low water mark flow rates of rivers and can reduce the basic level of the nappes that they drain, while increasing the regulatory role of these nappes: the contribution of underground water to keeping up the low water mark may increase. In other words droughts reduce the amount of water going in and increases the amount going out of waterways. Moreover, underground water can be further exploited to counter shortages of rainfall and water flows, with effects that would be more or less differed. The sensitivity of underground water to drought as well as its role in countering shortages depends to a large extent on the very varied hydrogeological conditions and situations in France.

Most free waterways resist annual droughts and are only sensitive to droughts over several years - whereas the captive nappes are insensitive, but their regulatory capacities are unequally exploited. 
1. Quelques exemples de variation de niveau de nappes souterraines libres en France de 1969 à 1988.

Profondeurs des niveaux en $\mathrm{m}$, échelles adaptées aux amplitudes.

Echelle de temps dilatée en 1988

1. Nappe de la craie à Preures (62).

2. Nappe de la craie à Barastre (62).

3. Nappe de la craie en Picardie à Feuquières-en-Vimeu (80).

4. Nappe de la craie en Normandie à Catenay (76).

5. Nappe du calcaire jurassique de La Plaine de Caen à Bretteville-l'Orgueilleuse (14).

6. Nappe de la Brie à Saint-Martin-Chennetron (77)

7. Nappe de la Beauce à Gidy (45).

8. Nappe du Plateau de Vaucluse à Gargas (84).

9. Nappe de la plaine d'Alsace à Rossfeld (67).

10. Nappe des Sables des Landes à Belin (33).

(Extraits du Bulletin « Situation hydrologique et prévisions de basses eaux »-Ed. BRGM).

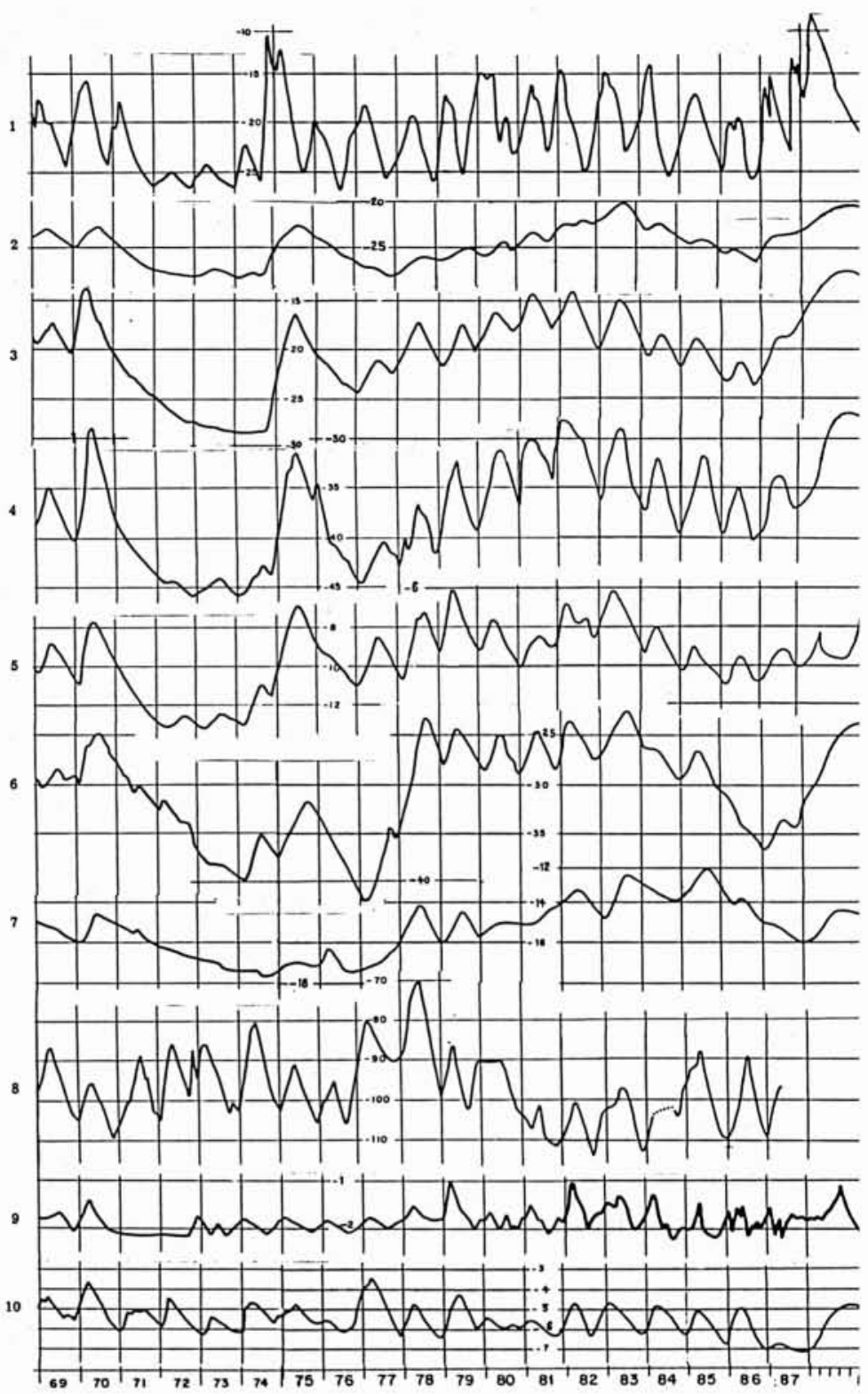

1) Variété des rapports entre flux et stock: En France, dans la plupart des aquifères à nappe libre d'extension régionale les volumes d'eau emmagasinés en moyenne sont au moins 5 à 10 fois supérieurs - parfois beaucoup plus aux volumes d'eau moyens annuels qu'ils reçoivent et qu'ils débitent. En revanche de nombreux aquifères alluviaux à débit élevé ont de faibles réserves, tandis qu'à l'inverse des nappes profondes, en général captives, des grands bassins sédimentaires sont des stocks considérables très faiblement renouvelés. Bien des aquifères étendus ont des réserves qui se chiffrent en milliards - parfois en dizaines de milliards - de $\mathrm{m}^{3}$ d'eau ; mais ces réserves sont extensives et représentent des hauteurs d'eau libre équivalentes de quelques mètres au plus, voire de quelques décimètres.
2) Variété des " ouvertures" des systèmes aquifères aux influences météoriques: selon que les nappes sont libres - et plus ou moins profondes - ou captives, elles sont très différemment réagissantes aux variations des conditions hydro-météoriques, que celles-ci soient ordinaires ou extrêmes; elles sont très inégalement réceptrices d'apports directs.

Lorsqu'elles le sont - cas général des nappes dites "phréatiques ", notons encore que cette alimentation par l'infiltration des eaux météoriques est fort discontinue : la plupart de ces nappes sont alimentées en France, en année " normale " entre octobre et mars ou avril, donc surtout par les pluies hivernales, en régime "normal » aussi bien qu'en régime sec. 
Pour mémoire rappelons qu'en France les alimentations des aquifères à nappe libre s'échelonnent en année moyenne suivant les régions, le climat et les conditions locales (types de sol, de couvert végétal...) entre 50 et $1000 \mathrm{~mm} / \mathrm{an}$ (équivalent, en flux continu par unité de surface, à une gamme de 2 à $30 \mathrm{l} / \mathrm{s}_{\mathrm{km}} \mathrm{km}^{2}$.

De plus ces apports sont très variables : 1 an sur 3 à 5 l'apport probable est inférieur à $50 \%$ de l'apport médian ; un apport quasi nul est probable 1 an sur 10 ( 1 sur 5 en régions méditerranéennes); entre les apports d'année sèche et d'année humide de fréquence 0,25 l'écart est du simple au double.

3) Variété des connexions avec les cours d'eau en surface: depuis les nappes souterraines très liées à des rivières (vallées fluviales) jusqu'aux nappes profondes totalement indépendantes, tous les cas de figures se présentent. Néanmoins, à des degrés divers, la plupart des nappes souterraines ont une fonction de " tampon » dans le système général de circulation des eaux, les nappes libres ayant le plus d'« inertie ». De ce fait leur débit - donc leur contribution aux cours d'eau, l'« écoulement souterrain " - est en général plus régulier que leur alimentation, $\mathrm{y}$ compris à l'échelle pluri-annuelle. En conséquence les «budgets" d'eau des nappes souterraines libres sont rarement en équilibre à l'échelle annuelle mais sont le plus souvent tantôt excédentaires tantôt déficitaires: ce qui se manifeste par des alternances de solde positif ou négatif des recharges et décharges annuelles (fig. I).

Le monde des eaux souterraines est ainsi un tel théâtre de variétés qu'il dissuade d'énoncer des généralités au sujet de ses réactions aux sécheresses.

\section{Sensibilités du régime des eaux souterraines aux sécheresses}

La variable d'état spatio-temporelle qui caractérise essentiellement le régime des eaux souterraines est leur potentiel hydraulique - altitude du niveau piézométrique — dont la distribution indique à tout moment l'état des stocks et (par leurs différences entre les divers points d'une nappe et ses limites émissives - les gradients de potentiel -) celui des flux locaux ou globaux: leur variation dans le temps traduit le déséquilibre entre les flux reçus et débités par l'aquifère considéré. Les niveaux piézométriques sont à la fois la variable la plus accessible à l'observation et la plus importante pour les exploitants d'eau souterraine. Ils constituent bien la variable sensible aux modifications des conditions extérieures, notamment aux variations d'apport et c'est sur eux que les conséquences de sécheresses peuvent être le plus manifestes.

En fonction des modalités d'alimentation et de « restitution " (par les émergences, le drainage par les rivières...) des aquifères évoqués plus haut, les sécheresses ont des répercussions très inégales sur le régime des nappes souterraines :

a) Les moins sensibles sont celles qui ne sont pas directement alimentées par les apports météoriques (nappes captives) et aussi celles de faible étendue dont les niveaux sont très soutenus par des conditions de potentiel imposées aux limites (cours ou plan d'eau de surface); dans le second cas les variations d'apport se traduisent assez rapidement par des variations de débit. Il s'agit de nappes à niveaux et à stocks peu variables. Exemples: nappes profondes des bassins de Paris et d'Aquitaine, grandes nappes alluviales liées à des fleuves (Alsace), mais aussi nappes libres drainées par des réseaux hydrographiques très denses.

b) Les plus sensibles sont les nappes libres alimentées essentiellement par les précipitations, non soutenues à leurs limites (débitant surtout par des sources) et de faible réserve (aquifère mince et peu capacitif) : nappes perchées locales, notamment en domaine à structure compartimentée (terrasses étagées, plateau karstique perché, nappes locales des terrains de socle cristallin fissuré...).

En somme les eaux souterraines les moins abondantes sont les plus sensibles aux sécheresses.

c) En situation intermédiaire se trouvent des nappes libres alimentées aussi principalement par les précipitations mais assez étendues et à réserves notables, à faible densité de drainage par les cours d'eau : par exemple les nappes de la craie ou d'autres aquifères sédimentaires de plaine (Beauce), les réservoirs karstiques étendus à ample zone noyée débitant par débordement (Vaucluse). Celles-ci sont surtout sensibles à des sécheresses pluri-annuelles.

La forte saisonnalité des phases d'alimentation des nappes libres (cas b et c ci-dessus) entraîne pour celles-ci deux sortes de sensibilité aux sécheresses:

— sensibilité aux sécheresses d'hiver : « déficit » d'alimentation par rapport à la moyenne, voire apport nul, qui se traduit par des recharges faibles ou nulles, notamment par des évolutions des niveaux en régime de tarissement pluriannuel (ainsi que du débit des sources);

- sensibilité différée aux sécheresses d'été et d'automne : l'appauvrissement des réserves d'eau du sol (réserve utile ou « RU » des agrologues...) a pour conséquence de retarder l'efficacité des pluies suivantes pour l'alimentation des nappes, la reconstitution des réserves du sol primant. Un certain déficit d'alimentation hivernale suivante peut en résulter même sans sécheresse.

En somme la sécheresse a un double effet sur le régime des nappes sensibles :

- elle atténue ou annule la recharge saisonnière :

par exemple, en 1976, il a été estimé que le "déficit d'alimentation " (par rapport à la moyenne) des nappes phréatiques a dépassé $50 \%$ sur près de la moitié de la France et $75 \%$ en plusieurs régions (Nord du bassin de Paris, Pays de la Loire, Bretagne, Midi) $\left({ }^{*}\right)$;

- elle augmente la durée des phases de décharge - suivant la dynamique interne de chaque système aquifère : les lois de tarissement qui lui sont propres - donc amplifie les baisses de niveau jusqu'à la phase de recharge suivante.

(*) Source : " La sécheresse au 31 mai 1976 », Secr. d'Etat à l'environnement et à la qualité de la vie. 


\section{Effet par l'aval}

Un effet plus accessoire, mais parfois non négligeable est indirect : c'est celui de la baisse de niveau d'eau superficielle imposant une condition de potentiel à la limite d'une nappe, en cas de tarissement prononcé. Le «niveau de base " qui commande la décharge de la nappe étant alors modifié, la baisse des niveaux est accentuée. Plus d'influence s'attache à un déplacement de limite parfois induit par les baisses dans le réservoir aquifère lui-même: l'«extinction» de source temporaire, la régression de la tête d'un cours d'eau permanent, mettent en jeu une nouvelle loi de tarissement de la nappe.

Néanmoins les variations de potentiel à une limite de ce type restent en général minimes par rapport à celles des potentiels distribués dans l'aquifère ; leur portée est localisée. De plus lorsqu'il s'agit de cours d'eau dont l'essentiel du débit en étiage provient du drainage d'un aquifère, une "boucle" interactive relie les tarissements décélérés du débit de l'un et des niveaux de l'autre (cf. infra 3)...

La sécheresse augmente-t-elle le "débit " des nappes souterraines - au sens de leurs dépenses, symétriquement à la diminution de leurs "recettes»-? En partie, mais seulement quant à la part de l'écoulement souterrain provenant du déstockage, et localement lorsque le niveau de base est abaissé. Mais non globalement: une nappe souterraine s'écoule en permanence, même et surtout en phase de recharge lorsque les potentiels internes sont au plus haut (hors du cas à inversion de flux à une limite : alimentation de nappe alluviale par un fleuve en crue...); elle débite davantage en période humide qu'en période sèche et la réduction progressive du débit sous l'effet de la décharge des potentiels "économise» la réserve. En somme la sécheresse favorise la vidange mais le tarissement prolongé qu'elle détermine diminue le flux sortant, de manière décélérée.

La sélection de variations de niveau piézométrique de nappes souterraines observées au cours des vingt dernières années en différentes régions de France, présenté fig. 1 , montre des exemples de la variété des sensibilités des régimes des nappes aux sécheresses saisonnières ou pluriannuelles. On remarque notamment :

- des exemples de recharge annuelle nulle ou minime de nappes libres étendues - 2 à 7 - en année sèche (1971, 1972, 1976, 1986) ;

- l'incidence de séquence d'années sèches (1971-1973) nettement plus ample que celle d'une année sèche isolée, même exceptionnelle (1976): exemples de tarissements pluri-annuels quasi ininterrompus en certains secteurs de nappe de la craie $(2,3,4)$ ou en Beauce (7);

- le contraste entre des nappes à régime essentiellement annuel, sans tendances pluri-annuelles marquées $(1,9,10)$ et des nappes où aux variations annuelles se superpose un régime pluri-annuel avec alternance de phases "déficitaires " (décharges supérieures aux recharges) et de phases excédentaires (recharges supérieures aux décharges); on note que les nappes phréatiques à niveau peu profond $(9$, 10) sont en général du premier type ; on note encore qu'une seule recharge annuelle peut compenser la décharge cumulée d'une séquence pluri-annuelle déficitaire de 3 à 4 années (exemples en 1975: 1 à 5);

- des exemples de nappes dont le régime est insensible aux sécheresses du fait de limites proches à potentiel imposé $(9,10)$.

\section{Contributions des eaux souterraines à l'atténuation des effets de la sécheresse}

Les aquifères formant les principaux sinon les seuls réservoirs naturels des systèmes d'eau continentaux - du moins hors des bassins dotés de glaciers ou de grands lacs... - ils assurent le plus généralement une fonction régulatrice spontanée, inter-saisonnière et interannuelle. On sait que l'essentiel du " débit de base » des cours d'eau correspond aux écoulements souterrains en phase de décharge. Ce qui vaut en étiage "normal " vaut d'autant plus en année sèche à étiage à la fois précoce et prolongé. La contribution des écoulements souterrains ne diminue ellemême à l'échelle annuelle que dans le cas d'aquifères de faible capacité (type b ci-dessus) et elle peut se réduire davantage si une succession d'années sèches survient. Mais la grande inertie de la plupart des aquifères libres les dotent d'une capacité régulatrice assez durable.

Dans un bassin donné la part de la composante relativement stable de l'écoulement total, en année moyenne comme en année sèche, est donc directement tributaire de l'importance et de l'abondance des aquifères à nappe libre connectés au réseau hydrographique, qui offrent en somme une " assurance/sécheresse ». Point n'est besoin d'épiloguer, à ce sujet, sur les contrastes entre la variabilité atténuée des écoulements de cours d'eau à bassin "tout aquifère " (exemples: Somme, I11) et celle beaucoup plus ample des écoulements de cours d'eau à bassin pauvre en réservoirs souterrains (exemples: Vilaine, Buech, HersMort). A cet égard le rôle des aquifères karstiques est moins efficace car leur écoulement souterrain est souvent assez variable lui-même, aussi sont-ils de moins bons régulateurs, notamment en cas de sécheresse.

A leur fonction régulatrice spontanée, toujours grâce à leur capacité lorsqu'elle est assez ample, les aquifères joignent un potentiel régulateur comme ressource, en offrant la possibilité de moduler leur exploitation: soit pour pallier les déficiences de précipitations pour les cultures par des irrigations de complément accrues, soit pour compenser des défaillances de ressources en eau de surface comme source d'approvisionnement, soit même pour renforcer artificiellement des débits d'étiage de cours d'eau. L'avantage offert par les eaux souterraines, comme ressource auto-régularisée dans une grande mesure, est déjà largement mis à profit en période courante ; il ne s'agit que d'amplifier cette utilisation en période de sécheresse. Cela requiert toutefois deux conditions :

- des conditions hydrogéologiques : l'aquifère doit avoir une capacité suffisante et les productivités locales ne doivent pas entraver son exploitation; de plus les réactions 
à des surproductions temporaires doivent être assez différées, afin que des impacts négatifs ne neutralisent pas les avantages ;

- des conditions techniques: un minimum de capacités d'exploitation excédentaires - non en service en année " normale "- est nécessaire. Si les équipements en place sont "saturés " en année ordinaire, la réalisation rapide d'ouvrages de captage complémentaire est malaisée (nonobstant la possibilité de modifier des priorités d'affectation de productions d'eau existantes...).

Par exemple, en France en 1976, on sait que sur les 310 interventions d'urgence réalisées avec l'aide des agences financières de bassin (dont 134 en Seine-Normandie), d'un montant total de $60,8 \mathrm{MF}, 144$ ont eu pour objet un " renforcement de ressource ", sur lesquelles $129(90 \%)$ ont concerné l'eau souterraine (forage, puits, captage de source...) $\left({ }^{*}\right)$.

Admettre le principe et faire en sorte que des ouvrages d'exploitation d'eau souterraine s'intègrent dans un plan de prévention contre les risques de sécheresse - donc qu'on ne vise pas leur rentabilité immédiate en année moyenne - paraît cependant plus difficile dans le contexte socio-économique et administratif français, que de réaliser des aménagements hydrauliques de surface pour la même fin (problèmes de maitrise d'œuvre, de propriété des sites, de répartition des coûts...). Pourtant le coût d'un barrage équivaut à celui de nombre de forages, du moins en investissement.

On doit cependant rappeler, que le Ministère de l'Agriculture, au titre de l'article 151 du Code rural, a engagé en 1978 un programme de "recherche d'eau " visant expressément les eaux souterraines et basé en priorité sur «la prospection de zones où les nappes sont encore peu connues et où la sécheresse de 1976 a révélée la vulnérabilité des alimentations actuelles " $\left.{ }^{* *}\right)$ (Circulaire du
13.12.1977). Sur ce programme de 109 opérations, les forages et les études de nappes ont représentés $18 \mathrm{MF}$ en $1^{\text {re }}$ tranche (C. BASALO, 1979).

Dans l'ensemble toutefois les équipements d'exploitation d'eau souterraine en service en France ne paraissent relativement adaptés à la fonction de surproduction temporaire en année de sécheresse exceptionnelle que pour l'utilisation agricole, là où l'irrigation par eau souterraine est dominante (Bassin de Paris notamment) et où l'accroissement de production dépend surtout des durées de pompage. On manque toutefois de statistiques probantes pour mesurer les surplus effectifs de prélèvements agricoles d'eau souterraine en année sèche. On a observé par contre qu'en 1976 les prélèvements d'eau souterraine pour l'alimentation en eau potable des collectivités (soumis à redevance par les agences financières de bassin) s'étaient accrus d'environ $5 \%$ en Seine-Normandie et $8,5 \%$ en LoireBretagne, par rapport à ceux des années voisines.

$\mathrm{Si}$ des équipements d'exploitation d'eau souterraine étaient plus développés pour mieux pallier aux incidences de la sécheresse, faudrait-il craindre, à l'inverse des risques de "surexploitation" de nappe souterraine? Sans les exclure, on peut penser que de tels risques ne pourraient procéder que soit de surestimation des capacités de certains aquifères locaux - et surtout en cas de sécheresse prolongée pluriannuelle - , soit d'une déviation d'utilisation des équipements mis en service régulièrement en année " normale" pour mieux les "valoriser "... Des opérations de surproduction temporaire devraient donc être accompagnées de suivi rigoureux des réactions des nappes et impliquerait une discipline des exploitants.

(*) M. DARgent, "La sécheresse exceptionnelle de 1976 ", $57^{\circ}$ Cong. AGHTM, Montreux, juin 1977.

$\left({ }^{* *}\right)$ En matière d'alimentation des communes rurales.

\section{En résumé :}

La plupart des nappes souterraines libres résistent à des sécheresses annuelles, leurs débits sont surtout sensibles à des sécheresses pluri-annuelles tandis que leurs niveaux ne fléchissent qu'assez loin de leurs limites à condition de potentiel; les nappes captives sont insensibles. Le jeu des capacités régulatrices des nappes libres contribue à atténuer les effets de sécheresse sur le régime des cours d'eau. Les réserves de beaucoup de nappes souterraines pourraient être davantage mises à contribution pour compenser les défaillances d'eau de surface et répondre aux demandes en eau en année sèche. 


\section{Repères bibliographiques}

BASAlo C. (1979). - Une conséquence de la sécheresse de 1976 : les 109 opérations "Recherches d'eau ". Travaux, janv., pp. 20-21, Paris.

BÉdıot G. (1985). - Faire face à la sécheresse. L'expérience française. La Houille Blanche, $\mathrm{n}^{\circ}$ 6-7, pp. 545-557, Grenoble.

Bodelle J., Margat J. (1980). - L'eau souterraine en France. Masson, 208 p., Paris.

Castany G., Margat J. (1977). - La sécheresse de 1976 et les eaux souterraines. AGHTM, $57^{\circ}$ Congrès, MontreuxLucerne. TSM $-L^{\prime} E a u, \mathrm{n}^{\circ} 10$, oct., pp. 409-412, Paris.

Delarozière-Bouillin O., Forkasiewicz J., Margat J. (1977). - Variations de l'alimentation des nappes en France: donnée importante pour la gestion des eaux souterraines. Coll. nat. " Eaux souterraines et approvisionnement en eau de la France ". BRGM, Nice, oct. 1977, T.I., pp. 203-222, Orléans.

MARGAT J. (1986). — "France " in "Ground Water in Europa ". UN/DTCD, Natural Resources/Water Serie, à paraître en 1989, New York.

Public. BRGM sous le titre "Abrégé sur les eaux souterraines de la France ", doc. 86 SGN 623 EAU, 61 p., Orléans.

Périodique. - "Situation hydrologique et prévisions de basses eaux ". Public. interministérielle trimest. 38 numéros parus depuis 1977, éd. BRGM. 